\title{
The scheduling of discriminative cues in successive discrimination learning
}

\author{
JOHN KOEHLER, JR. ${ }^{1}$ \\ INDIANAPOLIS CENTER OF INDIANA UNIVERSITY
}

\begin{abstract}
The proportion of negative cues and the frequency of switching the discriminative cues in the presentation sequence of the successive discrimination paradigm were treated in a 2 by 2 design, having nine rats per cell. In support of a suggestion by Hull, negative-topositive cue ratios larger than one increased discrimination accuracy following pretraining with the positive cue. To a lesser extent, the data also suggested that infrequent cue switches led to better discrimination than frequent ones, a finding noted to be consistent with the cue ratio outcome but in disagreement with the BushMosteller frequent cue switching principle.
\end{abstract}

\section{Introduction}

Conditioning a response to the positive cue to near asymptote in the prediscrimination period is reported to produce strong generalized response tendencies to the negative cue which then interfere with discrimination learning. Two somewhat conflicting hypotheses have been proposed concerning the conditions that would facilitate later discrimination learning. Based on his theoretical position, Hull (1952) has suggested that extinction of these generalization tendencies can be accelerated by increasing the number of negative cue presentations relative to the positive cue ones during training. In their discrimination learning model, Bush \& Mosteller (1951) have postulated a mathematical "discrimination" operator that applies only when the reward conditions change, which normally occurs when one cue is followed by the other cue in the presentation order during successive discrimination training. It is apparent, then, that their frequent cue switching principle runs counter to Hull's suggestion where an increase in the proportion of negative cue presentations will normally lead to a reduction in cue switching frequency over a given block of trials.

In order to evaluate cue sequence effects in successive discrimination with respect to these divergent theoretical predictions, the present study was designed to assess both cue switching frequency and negative-topositive cue ratios on discrimination performance after pretraining with the positive cue has taken place. Independent variation of these variables was achieved by inter-changing the positive and negative cues in the presentation sequence to give two levels of cue ratio for each amount of cue switching frequency.

\section{Method}

When water deprived for $22 \mathrm{hr}$, rats were trained to press a retractable, cylindrical bar for a dipper of water in a sound and light-proof cubicle. The training routine for each $S$ went as follows: (1) 7 days of gentling, (2)
3 days of dipper training, (3) 2 days of training to press the bar, (4) 20 positive cue-bar pairings on each of 10 days, (5) 7 days of discrimination training, and (6) a test day. On each discrimination training day, the series of 19 cue-bar pairings conformed to the following 2 by 2 design: 14 and 6 cue switches were combined with 12 and 7 , or 7 and 12, negative and positive cues. Group designations were: Group I with 14 switches, 12 - and 7 + cues; Group II with 6 switches, 12 - and 7 + cues; Group III with 14 switches, 7 - and $12+$ cues; and Group IV with 6 switches, 7 - and $12+$ cues. Three sequences were constructed for each cell of the design and rotated over successive discrimination training days. On the test day, a randomized sequence of 15 positive and 15 negative cue-bar pairings was used.

Nine rats were randomly assigned to each cell of the design from a group of 36 naive, male Sprague-Dawley albinos. Another 20 rats were discarded for reasons of apparatus failure or failure to train, but this loss was not differential over the treatment groups. At the start of training, Ss approximately ranged from 80 to 140 days old in each cell.

Presentation of the discriminative cues was accomplished by energizing a $7-1 / 2-w$ lamp with 30 or $90 \mathrm{v}$. The lamp was located behind a 1-in diameter Lucite disc which was flush with the wall about an inch above the bar. In a counterbalanced pattern, either 4 or 5 Ss in each cell were trained with the $30-$ or $90-v$ light as the positive cue and the other light as the negative cue.

The sequence of events on a particular cue-bar pairing was as follows: the lamp lit under the proper voltage; $5 \mathrm{sec}$. later, the bar was inserted into the cubicle; and following the bar-press response, the bar retracted, the lamp went off and a water dipper below the bar at floor level either refilled (positive cue) or remained inactive (negative cue). Insertion of the bar into the cubicle started a Standard Timer which stopped when the bar was depressed. Spacing between cue-bar pairings was 30,45 , or $60 \mathrm{sec}$. , rotated randomly so that the average interval was $45 \mathrm{sec}$. During discrimination training, if the bar was not pressed after $3 \mathrm{~min}$. in the presence of the negative cue, it was retracted; this time limit was reduced to $2 \mathrm{~min}$. on the test day.

\section{Results and Diseussion}

Latency of the bar press was taken as the measure of response strength to the discriminative cues. If the bar press had not occurred in the presence of the negative cue before the set time limit, the latency score was the value of the limit. To determine each S's discriminatory 


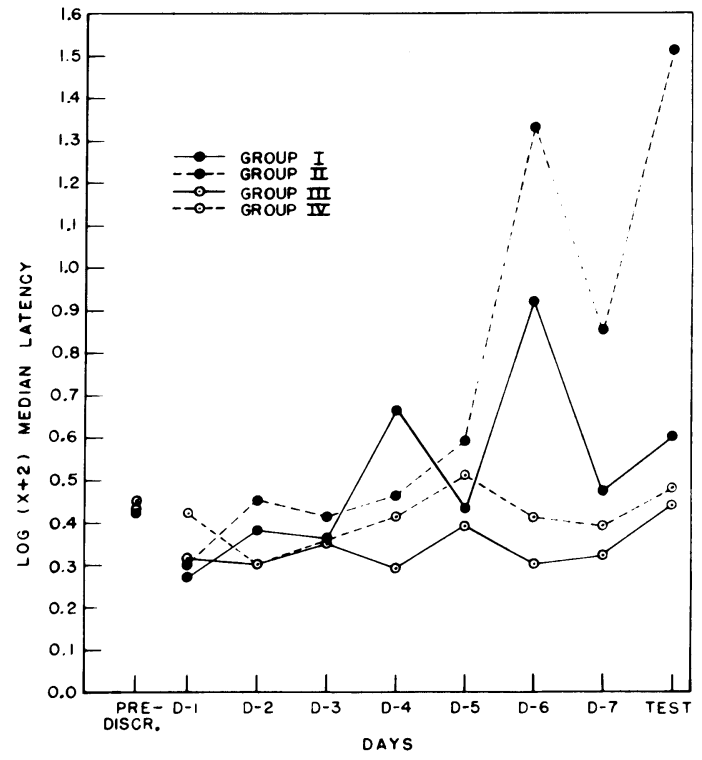

Fig. 1. Log $(X+2)$ median latency scores under each cue sequence combination plotted over the last prediscrimination day, 7 training days and the test day.

reaction, the median latency score to the positive cue was subtracted from the same score to the negative cue for each discrimination training day and the test day. A median latency score to the positive cue was also obtained for the last prediscrimination day.

Figure 1 plots the medians of these scores in logs for each treatment group. The near equivalence in response latency on the last prediscrimination day, as shown in the figure, was in agreement with the variance analysis of the median latency scores, which yielded $F$ ratios all less than one. From the figure, it is also apparent that discrimination training resulted in fairly distinct levels of performance among the four treatment groups. An analysis of variance which had (1) negativeto-positive cue ratio, (2) cue switching frequency, and (3) voltage of positive cue lamp as factors was performed on the test day scores after transformation to $\log (\mathrm{X}+3)$. The analysis revealed that Groups I and II, wh ve ratios were larger than one, were discriminating significantly better than the groups with ratios less than one, $F(1,28)=13.45, p<.01$. This finding agrees with Hull's supposition that the negative cue trial contributes more to discrimination learning than the positive cue trial in the case where the latter cue gives rise to strong generalized response tendencies to the former cue.

The cue switching frequency variable fell short of significance, $F(1,28)=4.01, p<.10$. But most interesting was finding that the order among the four groups was contrary to the prediction made from the Bush-Mosteller model (1951): Groups II and IV which received infrequent switches of the cues during training were discriminating better than their corresponding cue ratio groups, I and III, having frequent cue switches (See Fig. 1).

The analysis also found factor (3) to be insignificant, $F(1,28)=3.10, p<.10$, all pairwise interaction $F$ s very near one, and the 3-factor interaction insignificant, $F(1,28)=2.76, p<.25$. An examination of the untransformed data, however, suggested a possible switching frequency by cue ratio interaction effect. This effect appears in Fig. 1 even with the log transformation; Group II's discrimination learning rate tends to be faster than that of the other groups. This group was trained with infrequent cue switches and a negative-to-positive cue ratio greater than one: the interaction is consistent with Hull's suggestion for extinguishing cue generalization effects. Therefore, the outcome of the present study gives little support to the frequent cue switching principle as an important condition for successive discrimination learning following positive cue conditioning.

\section{Referemees}

Bush, R. R., \& Mosteller, F. A model for stimulus generalization and discrimination. Psychol. Rev., 1951, 58, 413-423.

Hull, C. L. A behavior system. New Haven: Yale University Press, 1952.

\section{Note}

1. Now at the University of Alabama. An earlier form of this paper was presented at the 1958 MPA meetings in Detroit. 